\title{
A New Method to Express Functional Permissibilities for LUT based FPGAs and Its Applications
}

\author{
Shigeru Yamashita, Hiroshi Sawada and Akira Nagoya \\ NTT Communication Science Laboratories \\ 2-2 Hikaridai, Seika-cho, Soraku-gun, Kyoto 619-02, JAPAN \\ \{ger, sawada, nagoya\}@cslab.kecl.ntt.jp
}

\begin{abstract}
This paper presents a new method to express functional permissibilities for look-up table (LUT) based field programmable gate arrays (FPGAs). The method represents functional permissibilities by using sets of pairs of functions, not by incompletely specified functions. It makes good use of the properties of LUTs such that their internal logics can be freely changed. The permissibilities expressed by the proposed method have the desired property that at many points of a network they can be simultaneously treated. Applications of the proposed method are also presented; a method to optimize networks and a method to remove connections that are obstacles at the routing step. Preliminary experimental results are given to show the effectiveness of our proposed method.
\end{abstract}

\section{Introduction}

Because of their low cost, re-programmability and rapid turnaround times, field programmable gate arrays (FPGAs) have emerged as an attractive means to implement low volume applications and prototypes[1]. FPGAs also offer the possibility to design digital systems that can be easily reconfigured. There are many types of commercially available FPGAs[1]. One particularly popular type, lookup table (LUT) based FPGAs, consist of an array of programmable logic blocks which contain LUTs and a programmable routing network to connect the LUTs. Each LUT can realize any boolean function with $m$ (typically 4 or 5) inputs.

The traditional design flow for LUT based FPGAs is as follows[1]. In the first step, a logic optimizer performs technology independent optimization $[2,3]$. Next, a technology mapper $[4,5,6,7,8,9,10,11]$ maps networks to LUTs. Finally, placement and routing are done. Networks are optimized in the first step using the number of literals for the cost function expected to be realized with gate arrays or standard cells. In the second step, most of the technology mappers start from multi-level networks whose nodes are represented by sum-of-product forms obtained in the first step. Thus LUT networks obtained by the technology mappers usually have some functional redundancies. On the other hand, some technology mappers $[7,9,11]$ directly generate LUT networks from primary output functions in terms of primary inputs represented by an ordered binary decision diagram (or simply BDD)[12]. These methods are not effected by intermediate sum-of-product forms and usually generate better LUT networks than the former technology mappers. However, they can not treat large networks because of limited BDD power. Large networks, therefore, must be divided and recombined to apply these methods. In such cases, there are some functional redundancies around the boundaries between divided networks. Either way, there are some functional redundancies in LUT networks after technology mapping. Such redundancies can be expressed by incompletely specified functions such as satisfiability don't cares (SDCs), observability don't cares (ODCs) [13] or Compatible Sets of Permissible Functions (CSPFs) [3]. CSPFs have been used to express redundancies and optimize LUT networks[14]. Although expressing redundancies by incompletely specified functions is very efficient for logic optimization[15], it does not utilize such flexibility as freely changing the internal logic of an LUT.

In this paper, we propose a new method to express functional permissibilities for LUT based FPGAs. The method utilizes not incompletely specified functions, but sets of pairs of functions. The sets are called "Sets of Pairs of Functions to be Distinguished (SPFDs)". SPFDs represent functional permissibilities utilizing properties of LUTs such that their internal logics can be changed, and are suitable for expressing functional permissibilities in LUT networks. SPFDs can be calculated as efficiently as CSPFs. Furthermore, the permissibilities expressed by SPFDs have the desired property of being able to be simultaneously treated at many points of a network as CSPFs (or compatible observability don't care sets). As an application of SPFDs, a method to change connections in LUT networks is proposed. This method can be applied to optimize LUT networks and to remove unroutable connections in the routing step.

This paper is organized as follows. In Section 2, we explain basic terminology and give an example of expressing don't care sets. In Section 3, the notion of SPFDs is introduced. The way to calculate SPFDs and their comparison with CSPFs are also described in Section 3. In Section 4, we discuss applications of SPFDs. Section 5 gives preliminary experimental results and our observations. Finally, Section 6 concludes this paper and mentions future work.

\section{Preliminaries}

\subsection{Terminology}

In this section, we provide the terminology for the rest of this paper. We treat loop-free multi-level combinational 


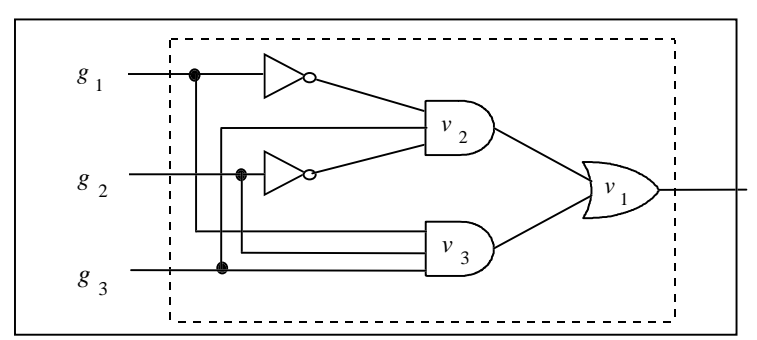

Figure 1: A part of a network

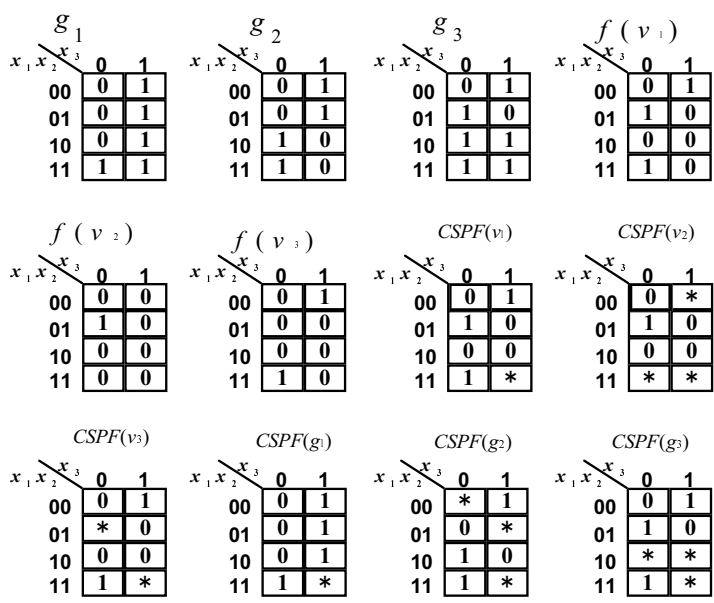

Figure 2: Calculation of CSPFs

networks that consist of LUTs and connections between them, and call such networks as LUT networks. The maximum number of inputs of an LUT is fixed (typically 4 or 5). Let $f\left(L_{i}\right)$ (or $f\left(c_{j}\right)$ ) be the logic function in terms of primary inputs realized at LUT $L_{i}$ (or connection $\left.c_{j}\right)$. The set of permissible functions[3] of an LUT (or a connection) is the set of functions; we can change the function realized at the LUT (or the connection) to a member of the set of the functions without changing the functionalities of the primary outputs of the network. CSPF [3] is one of the sets of permissible functions with the property that we can change functions of many LUTs to their CSPFs at the same time. The CSPF of an LUT (or a connection) is represented with an incompletely specified function whose values are 1,0 or $*$ (means don't care).

\subsection{Expressing Functional Permissibilities by CSPFs}

Here, how to express functional permissibilities by CSPFs is explained by the following example.

Figure 1 shows a part of a network. Let $v_{1}$ be an output gate of the network and $g_{1}, g_{2}$ and $g_{3}$ be intermediate functions that are expressed by the primary inputs $x_{1}, x_{2}$ and $x_{3} . \quad g_{1}, g_{2}$ and $g_{3}$ are shown in Fig. 2. Here, the functions realized at gates $v_{1}, v_{2}$ and $v_{3}$ are $f\left(v_{1}\right), f\left(v_{2}\right)$ and $f\left(v_{3}\right)$ in Fig. 2, respectively. If the bit of $f\left(v_{1}\right)$ is don't care when $\left(x_{1}, x_{2}, x_{3}\right)$ is $(1,1,1)$ (external don't care), the CSPF of $v_{1}$ is expressed as "CSPF $\left(v_{1}\right)$ " in Fig. 2. If a bit of $f\left(v_{1}\right)$ is 1 , the corresponding bit of either $f\left(v_{2}\right)$ or $f\left(v_{3}\right)$ must be 1 , but the other's is don't care since $v_{1}$ is an OR gate. For example, when $\left(x_{1}, x_{2}, x_{3}\right)$ is $(0,0,1)$, the bit of $f\left(v_{3}\right)$ is 1 ; therefore, the bit of $f\left(v_{2}\right)$ is don't care. In this way, the CSPFs of input functions of $v_{1}$ are calculated as "CSPF $\left(v_{2}\right)$ " and "CSPF $\left(v_{3}\right)$ " in Fig. 2. For other kinds of gates, the CSPFs of the gates' inputs are calculated by the same notion. The functional permissibilities expressed by CSPFs are calculated from primary outputs in the direction of primary inputs of a network. If a gate has more than two fanouts, the CSPF of the gate is calculated by the intersection of the fanouts' CSPFs. In this example, the CSPFs of the connections concerning $g_{1}, g_{2}$ and $g_{3}$ are calculated as "CSPF $\left(g_{1}\right)$ ", "CSPF $\left(g_{2}\right)$ " and " $C S P F\left(g_{3}\right)$ " in Fig. 2, respectively. " $C S P F\left(g_{1}\right)$ ", " $C S P F\left(g_{2}\right)$ " and "CSPF $\left(g_{3}\right)$ " will be compared with our new expression in Section 3.

\section{Functional Permissibility Expression for LUT based FPGAs}

3.1 Sets of Pairs of Functions to be Distinguished

The CSPF of $L_{i}$ is represented by an incompletely specified function. This means that the alternative functions for $f\left(L_{i}\right)$ must be 1 or 0 when the CSPF is 1 or 0 , respectively. Such expression of the conditions of alternative functions is very useful in networks where the internal logic of each node is fixed. In an LUT network, however, the internal logic of each node can be changed; therefore, another method can be used for expressing the conditions of alternative functions. To explain such a method, some definitions are introduced here.

Definition 1 A function $f$ is said to distinguish a pair of functions $g_{1}$ and $g_{2}$ if either one of the following two conditions is satisfied.

condition $1\left(f=1\right.$ when $\left.g_{1}=1\right)$ and $(f=0$ when $\left.g_{2}=1\right)$.

condition $2\left(f=0\right.$ when $\left.g_{1}=1\right)$ and $(f=1$ when $\left.g_{2}=1\right)$.

Note that $\left(g_{1} \cdot g_{2}\right)$ must be the function that is constantly 0 .

For example, function $f$ (in Fig. 3) distinguishes a pair of functions $f_{1 a}$ and $f_{1 b}$ (in Fig. 3) because $f=1$ when $f_{1 a}=1$, and $f=0$ when $f_{1 b}=1 . f$ also distinguishes a pair of functions $f_{2 a}$ and $f_{2 b}$.

Definition 2 A set of pairs of functions $\left\{\left(f_{1 a}, f_{1 b}\right)\right.$, $\left.\left(f_{2 a}, f_{2 b}\right), \cdots,\left(f_{n a}, f_{n b}\right)\right\}$ represents the conditions of functions such that the functions must distinguish $f_{i a}$ and $f_{i b}$ for each pair $\left(f_{i a}, f_{i b}\right)$ in the set.

For example, function $f$ (in Fig. 3) satisfies the conditions represented by $\left\{\left(f_{1 a}, f_{1 b}\right),\left(f_{2 a}, f_{2 b}\right)\right\}$ because $f$ distinguishes $f_{1 a}$ and $f_{1 b}$, and $f_{2 a}$ and $f_{2 b}$. 


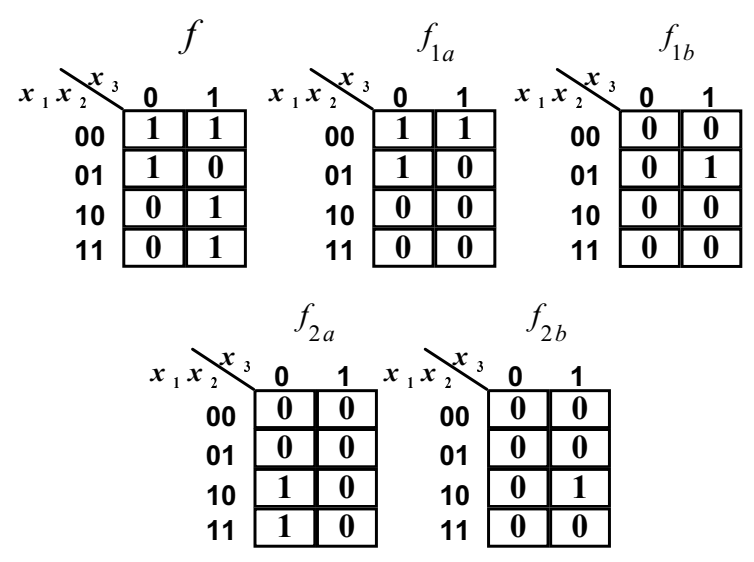

Figure 3: Functions to be distinguished

In LUT networks, we propose using a set of pairs of functions to represent the conditions of alternative functions instead of incompletely specified functions and introduce the following definition.

Definition 3 A set of pairs of functions that represents the conditions of the alternative functions for $f\left(L_{i}\right)$ (or $f\left(c_{j}\right)$ ) is called the "Set of Pairs of Functions to be Distinguished (SPFD)" of $L_{i}$ (or $c_{j}$ ). If a function $g$ satisfies the conditions represented by an SPFD, $g$ is said to "satisfy" the SPFD.

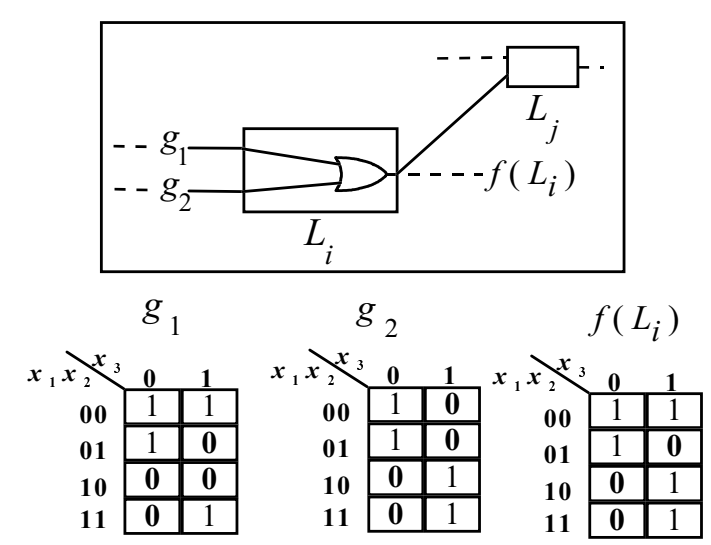

Figure 4: An LUT in a network

An intuitive explanation of SPFDs is given as follows. In Fig. 4, LUT $L_{i}$ has two inputs whose functions are $g_{1}$ and $g_{2}$, which are shown in Fig. 4. The internal logic of $L_{i}$ is OR; therefore, $f\left(L_{i}\right)$ is expressed as shown in Fig. 4. If $f\left(L_{i}\right)$ is used as an input of another LUT $L_{j}$, the role of $f\left(L_{i}\right)$ is to distinguish the two functions $f_{a}$ and $f_{b}$, which are expressed as shown in Fig. 5. $f_{a}$ and $f_{b}$ are the ON-set and the OFF-set of $f\left(L_{i}\right)$, respectively. Therefore, the SPFD of $L_{i}$ is $\left\{\left(f_{a}, f_{b}\right)\right\}$. Only $f\left(L_{i}\right)$ and $\overline{f\left(L_{i}\right)}$ satisfy the SPFD of $L_{i}$. For example, $\overline{f\left(L_{i}\right)}$ distinguishes $f_{a}$ and $f_{b}$ because $\overline{f\left(L_{i}\right)}$ is 1 when $f_{b}$ is 1 and $\overline{f\left(L_{i}\right)}$ is 0 when $f_{a}$ is 1 . If $f\left(L_{i}\right)$ is changed to $\overline{f\left(L_{i}\right)}$, we only modify the internal logic of $L_{j}$ to negate the input function from $L_{i}$.

$g_{1}$ (in Fig. 4) can distinguish $g_{1 a}$ and $g_{1 b}$ (in Fig. 5) which are the ON-set and the OFF-set of $g_{1}$, respectively. If the internal logic of $L_{i}$ can be freely changed, $f\left(L_{i}\right)$ can be changed to distinguish functions that can be distinguished by using both $g_{1}$ and $g_{2}$ (how to change $f\left(L_{i}\right)$ is shown in Section 4.1). Thus, if the SPFD of $L_{i}$ and function $g_{1}$ are not changed, $g_{2}$ must distinguish $g_{2 a}$ and $g_{2 b}$; therefore, the SPFD of the input connection concerning $g_{2}$ is $\left\{\left(g_{2 a}, g_{2 b}\right)\right\}$. This is because the conditions expressed by $\left\{\left(f_{a}, f_{b}\right)\right\}$ are the same as those expressed by $\left\{\left(g_{1 a}, g_{1 b}\right),\left(g_{2 a}, g_{2 b}\right)\right\}$. This is an intuitive way to calculate the SPFD of $g_{2}$. The formal way to calculate SPFDs is shown in Section 3.2.

Function $g_{2}^{\prime}$ in Fig. 5 distinguishes $g_{2 a}$ and $g_{2 b}$; therefore, $g_{2}$ can be replaced with $g_{2}^{\prime}$. If $g_{2}$ is replaced with $g_{2}^{\prime}$, the internal logic of $L_{i}$ must be modified to $\left(g_{1}+\overline{g_{2}^{\prime}}\right)$. How to modify the internal logic of an LUT is shown in Section 4.1.
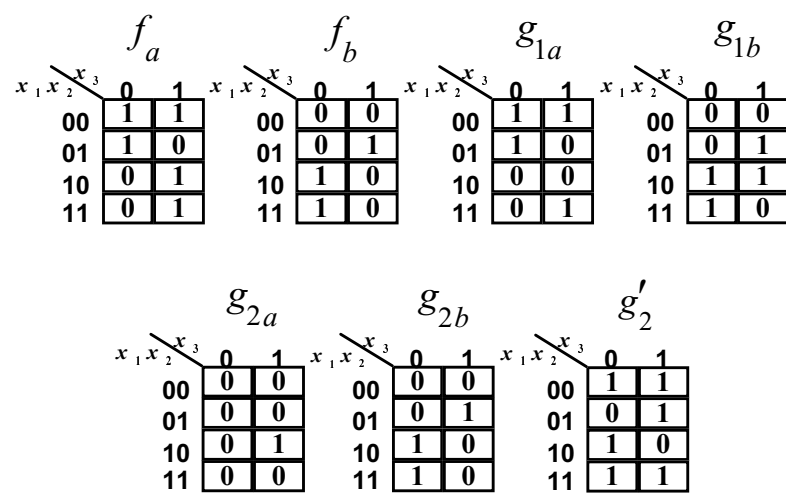

Figure 5: Functions in SPFDs

In this example, the permissibility of $g_{2}$ expressed by the SPFD is the same as that expressed by the CSPF. In more complicated cases, however, the permissibilities expressed by SPFDs are different from those expressed by CSPFs, and this is shown in Section 3.2.

\subsection{Calculating SPFDs}

3.2.1 Calculating the SPFDs of the Input Connections of an LUT

The procedure to calculate the SPFDs of the input connections of LUT $L$ is formally stated as follows. $L$ has $n$ input connections $\left(c_{1}, \cdots, c_{n}\right)$, and the functions realized 
at $\left(c_{1}, \cdots, c_{n}\right)$ are $\left(g_{1}, \cdots, g_{n}\right)$, respectively. Let the SPFD of $L$ be $\left\{\left(f_{1}, f_{0}\right)\right\}$, which has already been calculated.

Below, a function $f$ is said to be included in a function $g$ if $f \cdot \bar{g}$ is the function that is constantly 0 . Initially, let the SPFDs of $\left(c_{1}, \cdots, c_{n}\right)$ be empty.

step 1 Calculate $2^{n}$ logical products for all possible combinations of $\left(g_{1}, \cdots, g_{n}\right)$, where each $g_{i}$ is negated or not (e.g., $\left(\overline{g_{1}} \cdot \overline{g_{2}} \cdot, \cdots, \cdot \overline{g_{n}}\right),\left(g_{1} \cdot g_{2} \cdot, \cdots, \cdot g_{n}\right)$, and so on). Let these products be $\left(b_{0} \cdots 0, \cdots, b_{1} \cdots 1\right)$, where the index of $b_{k}$ is an $n$ bits binary number that satisfies the following condition.

- The i-th bit (from the left) of $k$ is 0 or 1 , depending on whether $g_{i}$ is negated or not in $b_{k}$.

For example, when $n=3, b_{011}=\overline{g_{1}} \cdot g_{2} \cdot g_{3}$.

step 2 For all $b_{i}$, calculate $a_{i}=b_{i} \cdot\left(f_{1}+f_{0}\right)$.

step 3 From $\left(a_{0 \cdots 0}, \cdots, a_{1 \cdots 1}\right)$, select all of the functions that are included in $f_{1}$ and not constantly 0 . Let the set of these functions be $F 1$. From $\left(a_{0 \cdots 0}, \cdots, a_{1 \cdots 1}\right)$, select all of the functions that are included in $f_{0}$ and not constantly 0 . Let the set of these functions be $F 0$.

step 4 Calculate the cartesian product $F=F 1 \times F 0$.

step 5 Select an element in $F$ as $\left(a_{i}, a_{j}\right)$ one by one, and go to step 6. If there is no element to select, halt.

step 6 If the different bits of $i$ and $j$ are the $k_{1}, k_{2}, \cdots, k_{s^{-}}$ th bits (from the left), select an arbitrary $k_{t}$ from them, add $\left(a_{i}, a_{j}\right)$ to the SPFD of $c_{k_{t}}$, and go to step 5 .

If the SPFD of $L$ has more than two elements, the above procedure is applied for all elements in the SPFD of $L$, and the SPFD of $c_{i}$ is calculated by the union of all the calculated SPFDs for $c_{i}$. Although the procedure consumes $O\left(2^{2 n}\right)$ time for the worst case where $n$ is the number of inputs of an LUT (even if the calculation time of logic functions is thought to be constant), in practice it does not consume so much time because $n$ is typically a small number (4 or 5). Therefore, this procedure is very suitable for LUT networks whose nodes have a small number of fanins.

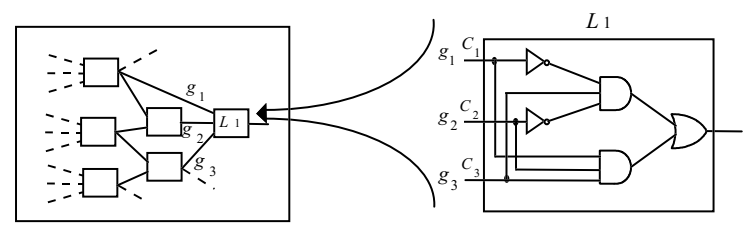

Figure 6: An LUT network

The procedure is explained using the following example. Figure 6 shows an LUT network. LUT $L_{1}$ has three input connections $c_{1}, c_{2}$ and $c_{3}$, and the functions realized at $c_{1}, c_{2}$ and $c_{3}$ are $g_{1}, g_{2}$ and $g_{3}$, respectively. $g_{1}, g_{2}$ and $g_{3}$ are shown in Fig. 2. The internal logic of $L_{1}$ is expressed as $\left(g_{1} \cdot g_{2} \cdot g_{3}+\overline{g_{1}} \cdot \overline{g_{2}} \cdot g_{3}\right)$ (shown in Fig. 6). The SPFD of $L_{1}$ has already been calculated as $\left\{\left(f_{1}, f_{0}\right)\right\}$, where $f_{1}$ and $f_{0}$ are the ON-set and the OFF-set of "CSPF( $\left.v_{1}\right)$ " shown in Fig. 2. $f_{1}$ and $f_{0}$ are shown in Fig. 7. The internal logic of $L_{1}$ has the same functionalities as the network shown in Fig. 1. Therefore, the conditions of this example are the same as those of the example in Section 2.2. Here, the SPFDs of $c_{1}, c_{2}$ and $c_{3}$ are calculated as follows.
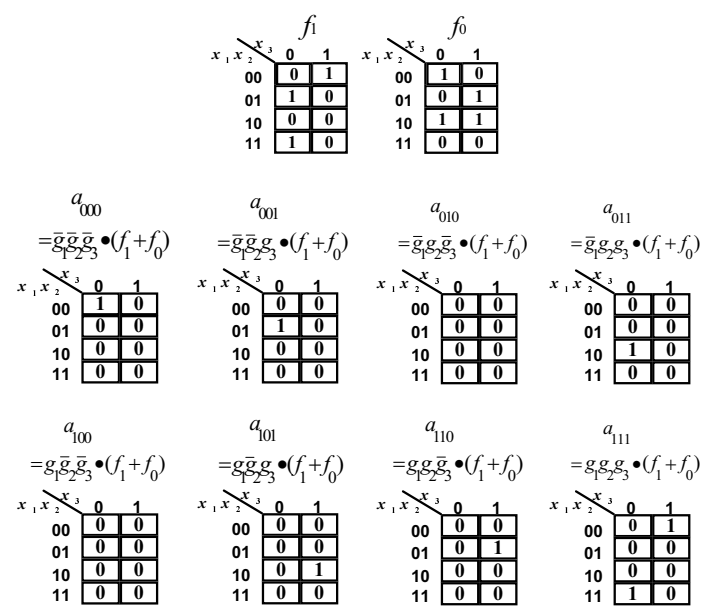

Figure 7: Calculating SPFDs (1)

step 1 Calculate $2^{3}$ logical products for all possible combinations of $g_{1}, g_{2}$ and $g_{3}$, where each $g_{i}$ is negated or not (e.g., $b_{000}=\overline{g_{1}} \cdot \overline{g_{2}} \cdot \overline{g_{3}}, b_{111}=g_{1} \cdot g_{2} \cdot g_{3}$, and so on).

step 2 For $\left(b_{000}, \cdots, b_{111}\right)$, calculate $a_{i}=b_{i} \cdot\left(f_{1}+f_{0}\right)$. $\left(a_{000}, \cdots, a_{111}\right)$ are shown in Fig. 7. This step removes don't care bits of the SPFD of $L_{1}$ from $b_{i}$.

step 3 Since $a_{001}$ and $a_{111}$ are included in $f_{1}$, let $F 1$ be $\left\{a_{001}, a_{111}\right\}$. Since $a_{000}, a_{011}, a_{101}$ and $a_{110}$ are included in $f_{0}$, let $F 0$ be $\left\{a_{000}, a_{011}, a_{101}, a_{110}\right\}$. There is no need to consider $a_{010}$ and $a_{100}$ because they are functions that are constantly 0 .

step 4 The set $F$ is calculated from the cartesian product $F 1 \times F 0 . F 1, F 0$ and $F$ are shown in Fig. $8 . F$ is a set of pairs of functions that must be distinguished by any one of the input functions of $L_{1}$. At step 5 and step 6 , the pairs in $F$ are divided into the SPFDs of the input connections of $L_{1}$.

step 5 For the first element in $F$ (i.e., $\left(a_{001}, a_{000}\right)$ ), go to step 6.

step 6 The third bits of (001) and (000) are different from each other, which means that $g_{3}$ can distinguish $a_{001}$ and $a_{000}$. This is because the difference between 
$\left(\overline{g_{1}} \cdot \overline{g_{2}} \cdot g_{3}\right)$ and $\left(\overline{g_{1}} \cdot \overline{g_{2}} \cdot \overline{g_{3}}\right)$ is whether $g_{3}$ is negated or not. Therefore, $\left(a_{001}, a_{000}\right)$ is added to the SPFD of $c_{3}$.

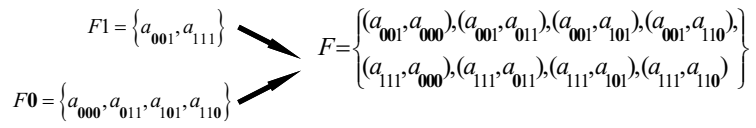

Figure 8: Calculating SPFDs (2)

For the remaining elements in $F$, step 5 and step 6 are done in the same way. For $\left(a_{111}, a_{000}\right)$, all bits of (111) and (000) are different, which means that all of $g_{1}, g_{2}$ and $g_{3}$ can distinguish $a_{111}$ and $a_{000}$. Therefore, $\left(a_{111}, a_{000}\right)$ can be added to any one of the SPFDs of $c_{1}, c_{2}$ or $c_{3}$ (this selection corresponds to the selection of $k_{i}$ from $k_{1}, k_{2}, \cdots, k_{s}$ at step 6 in the above procedure). If $c_{1}$ is selected in such cases, the SPFDs of $c_{1}, c_{2}$ and $c_{3}$ are calculated as " $\operatorname{SPFD}\left(c_{1}\right)$ ", "SPFD $\left(c_{2}\right)$ " and "SPFD $\left(c_{3}\right)$ " in Fig. 9. For example, the conditions of alternative functions for $g_{1}$ are represented by the SPFD of $c_{1}$ as follows.

- The bits of $g_{1}$ corresponding to the bits of " $A$ " and " $\bar{A}$ " in "Conditions by $S P F D\left(c_{1}\right)$ " (in Fig. 9) must be different from each other.

- The bits of $g_{1}$ corresponding to the bits of " $B$ " and " $\bar{B}$ " in "Conditions by $S P F D\left(c_{1}\right)$ " (in Fig. 9) must be different from each other.

Thus, the bits of either " $A$ " or " $\bar{A}$ " must be 1 , but the bits of the other must be 0 . There are two assignments of 1 and 0 to " $A$ " and " $\bar{A}$ ". In the same way, there are two assignments of 1 and 0 to " $B$ " and " $\bar{B}$ ". There are two assignments of 1 and 0 to " $*$ " in "Conditions by $\operatorname{SPFD}\left(c_{1}\right)$ " in Fig. 9. Therefore, the number of functions that satisfy the SPFD of $c_{1}$ is 8. The CSPF of $c_{1}$ is shown as "CSPF $\left(c_{1}\right)$ " in Fig. 9 (It is the same as "CSPF( $\left.g_{1}\right)$ " in Fig. 2). The number of functions that satisfy " $C S P F\left(c_{1}\right)$ " is 2 because the CSPF has one don't care bit. In this example, we can find more alternative functions for $g_{1}$ using SPFDs than using CSPFs. Comparisons of the SPFDs and the CSPFs of $c_{1}, c_{2}$ and $c_{3}$ are shown in Fig. 9. In this example, the internal logic of $L_{1}$ is not redundant. If the internal logic of an LUT is redundant, the difference between SPFDs and CSPFs becomes larger.

\subsubsection{Calculating the SPFD of an LUT}

The SPFD of LUT $L_{i}$ is obtained by the union of all the SPFDs of the output connections of $L_{i}$. For example, if $L_{i}$ has two fanouts $c_{1}$ and $c_{2}$, and the SPFDs of $c_{1}$ and $c_{2}$ are $\left\{\left(f_{11}, f_{10}\right)\right\}$ and $\left\{\left(f_{21}, f_{20}\right)\right\}$ respectively, the SPFD of $L_{i}$ is calculated as $\left\{\left(f_{11}, f_{10}\right),\left(f_{21}, f_{20}\right)\right\}$ unless $\left(f_{11}, f_{10}\right)$ and $\left(f_{21}, f_{20}\right)$ are the same. There are some cases where the number of elements in the SPFD of $L_{i}$ obtained by such a calculation becomes too large. In such cases, the SPFD

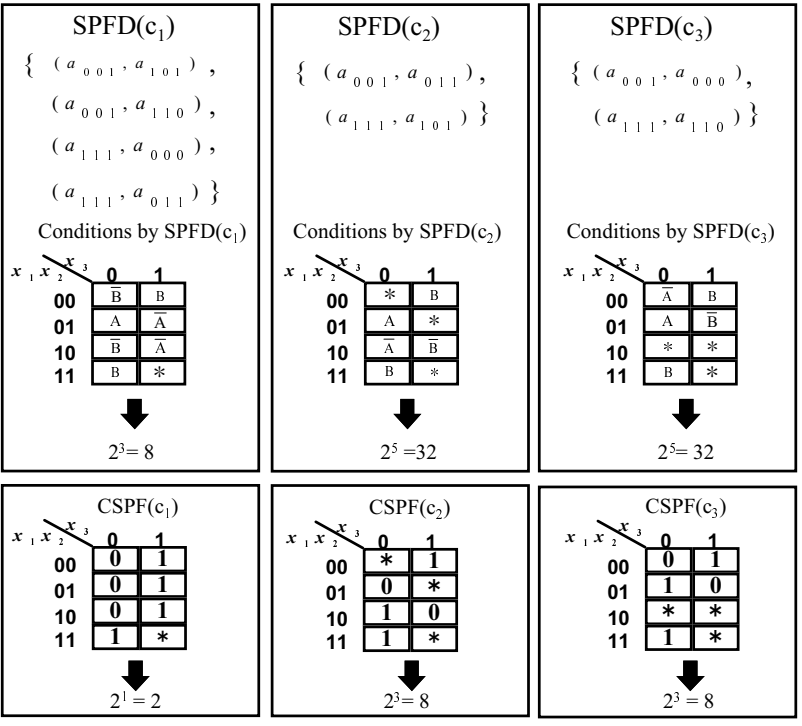

Figure 9: Comparisons of SPFDs with CSPFs

of $L_{i}$ can be filtered to have less elements. For example, the SPFD of $L_{i}$ in the above example can be filtered to be $\left\{\left(f_{11}+f_{21}, f_{10}+f_{20}\right)\right\}$ in the following cases (we can also do the same type of filtering in other cases).

- when $f_{11}$ and $f_{21}$ are both included in $f\left(L_{i}\right)$, and $f_{10}$ and $f_{20}$ are both included in $\overline{f\left(L_{i}\right)}$.

- when $f_{11}$ and $f_{21}$ are both included in $\overline{f\left(L_{i}\right)}$, and $f_{10}$ and $f_{20}$ are both included in $f\left(L_{i}\right)$.

Note that $f_{11}, f_{10}, f_{21}$ and $f_{20}$ are always included in either $f\left(L_{i}\right)$ or $\overline{f\left(L_{i}\right)}$. By this filtering, the number of elements in SPFDs becomes smaller, which contributes toward reducing the calculation time.

\section{Applications of SPFDs}

In this section, we discuss about applications of SPFDs. SPFDs at many points of a network can be simultaneously treated as CSPFs. Therefore, SPFDs can be used in the same way as CSPFs.

\subsection{Changing Connections Using SPFDs}

In LUT networks, we can exchange connection $c_{i}$ with the output of $L_{j}$, if $f\left(L_{j}\right)$ satisfies the SPFD of $c_{i}$.

In the example mentioned in Section 3.2.1, the conditions represented by the SPFD of $c_{3}$ are expressed as "Conditions by $S P F D\left(c_{3}\right)$ " in Fig. 9. $g_{3}^{\prime}$ shown in Fig. 10 satisfies the conditions because the bits of $g_{3}^{\prime}$ corresponding to the bits of " $A$ " and " $\bar{A}$ " in "Conditions by $S P F D\left(c_{3}\right)$ " are 0 and 1 , and the bits of $g_{3}^{\prime}$ corresponding to the bits of " $B$ " and " $\bar{B}$ " are 1 and 0 . Therefore, if LUT $L_{j}$ realizes function $g_{3}^{\prime}, c_{3}$ can be replaced with the output of $L_{j}$. If CSPFs are used, $c_{3}$ can not be replaced 


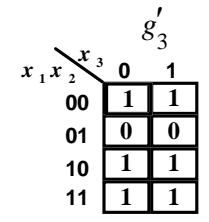

Figure 10: An alterative function for $g_{3}$

because $g_{3}^{\prime}$ does not satisfy "CSPF $\left(g_{3}\right)$ " in Fig. 2. When $c_{3}$ is replaced with the output of $L_{j}, f\left(L_{1}\right)$ is changed and does not satisfy the SPFD of $L_{1}$ anymore. Therefore, the internal logic of $L_{1}$ must be modified as follows so that $f\left(L_{1}\right)$ still satisfies the SPFD of $L_{1}$. Observing $F 1$ and $F 0$ obtained at step 3 in the example of calculating the SPFDs of input connections of $L_{1}$ in Section 3.2.1 $\left(F 1=\left\{a_{001}, a_{111}\right\}, F 0=\left\{a_{000}, a_{011}, a_{101}, a_{110}\right\}\right)$, we can see that $f\left(L_{1}\right)$ must satisfy the following two conditions.

condition $1 f\left(L_{1}\right)=1$ when $a_{001}=1$, and $f\left(L_{1}\right)=0$ when $\left(a_{000}=1, a_{011}=1, a_{101}=1\right.$ and $\left.a_{110}=1\right)$.

condition $2 f\left(L_{1}\right)=1$ when $a_{111}=1$, and $f\left(L_{1}\right)=0$ when $\left(a_{000}=1, a_{011}=1, a_{101}=1\right.$ and $\left.a_{110}=1\right)$.

The internal logic of $L_{1}$ must be modified so that $f\left(L_{1}\right)$ satisfies the above two conditions. $\overline{g_{2}}=1$ when $a_{001}=1$, and $\overline{g_{2}}=0$ when $\left(a_{011}=1\right.$ and $\left.a_{110}=1\right) \cdot \overline{g_{3}^{\prime}}=1$ when $a_{001}=1$, and $\overline{g_{3}^{\prime}}=0$ when $\left(a_{000}=1\right.$ and $\left.a_{101}=1\right)$. Therefore, the function $\left(\overline{g_{2}} \cdot \overline{g_{3}^{\prime}}\right)$ satisfies condition 1 . In the same way, the function $\left(g_{1} \cdot g_{2} \cdot g_{3}^{\prime}\right)$ satisfies condition 2. Therefore, the function $\left(g_{1} \cdot g_{2} \cdot g_{3}^{\prime}+\overline{g_{2}} \cdot \overline{g_{3}^{\prime}}\right)$ satisfies the above two conditions, which is the modified internal logic of $L_{1}$.

The procedure to modify the internal logic of LUT $L$ is formally stated as follows. $L$ has $n$ input connections whose functions are $\left(g_{1}, \cdots, g_{n}\right)$. Note that $\left(g_{1}, \cdots, g_{n}\right)$ may be changed by replacing connections. Let $F 1$ and $F 0$ be the sets obtained at step 3 of the procedure to calculate the SPFDs of input connections of $L$ (mentioned in Section 3.2.1). The modified internal logic is calculated as a sumof-product form, and the $\mathrm{i}$-th product is $l_{i}$. Initially, let all $l_{i}$ be the function that is constantly 1 .

step 1 Select the i-th element in $F 1$ as $a_{i}$, and go to step 2. If there is no element to select, halt.

step 2 Select an element in $F 0$ as $a_{j}$ one by one, and go to step 3 . If there is no element to select, go to step 1 .

step 3 From $\left(g_{1}, \cdots, g_{n}\right)$, select the function that distinguishes $a_{i}$ and $a_{j}$ as $g_{k}$, and go to step 4.

step 4 If $g_{k}$ is 1 when $a_{i}$ is 1 , modify $l_{i}$ to $\left(l_{i} \cdot g_{k}\right)$. If $g_{k}$ is 0 when $a_{i}$ is 1 , modify $l_{i}$ to $\left(l_{i} \cdot \overline{g_{k}}\right)$. go to step 2 .

Finally, the modified logic is obtained as $\left(l_{1}+l_{2}, \cdots,+l_{m}\right)$, where $m$ is the number of elements in $F 1$.

The output functions of the LUTs that are the transitive fanouts of $L_{1}$ may also change. The internal logics of such LUTs must be changed in the same way.

\subsection{Optimization Using SPFDs}

The following procedure optimizes an LUT network using SPFDs.

step 1 Calculate the SPFDs of all LUTs and connections in the network.

step 2 Select a connection as $c_{i}$ one by one, and go to step 3 . If there is no connection to select, halt.

step 3 If the SPFD of $c_{i}$ is empty, remove $c_{i}$ and go to step 5. Otherwise, go to step 4.

step 4 If $c_{i}$ can be replaced with the output of $L_{j}$, replace it and go to step 5. Otherwise, go to step 2.

step 5 If the logic functions of some LUTs are changed owing to removing or replacing $c_{i}$ with the output of $L_{j}$, change the internal logics of such LUTs properly (by the method mentioned in Section 4.1). Go to step 2 .

The order to select $c_{i}$ in step 2 and the order to select $L_{j}$ in step 4 are based on heuristics. For example, if the optimized networks should have lower levels, the LUT whose number of levels is the smallest is selected as $L_{j}$ in step 4. If the optimized networks should have less LUTs, the output of the LUT that has one fanout is selected first as $c_{i}$ in step 2. This is because if the output of the LUT that has one fanout is replaced with another LUT's output, the LUT can be immediately removed.

\subsection{Removal of Unroutable Connections Using SPFDs}

Because of limited routing resources in FPGAs, automatic routing may fail in a congested area, even though routing resources are available in a non-congested area. In such a case, the design is usually modified manually and the routing is tried again. We propose removing unroutable connections or replacing them with other connections by using our method mentioned in Section 4.1, and routing again automatically. This approach raises the possibility of successful automatic routing.

\section{Experimental Results}

We have implemented the methods presented here and performed preliminary experiments on MCNC[16] benchmark circuits. BDD was used for representing functions, and the maximum number of usable BDD nodes was limited to $1,000,000$. Therefore, some large circuits, e.g., $\mathrm{C} 3540$, C7552, C2670, etc., could not be treated. In the experiments, a 5-input LUT architecture was assumed. The SIS (A System for Sequential Circuit Synthesis of UC Berkeley) technology mapper commands were used to generate initial networks, i.e., eliminate 2 , gkx -ac, simplify -d, xl_part_coll -m -g 2, xl_coll_ck, xl_partition -m, simplify, xl_imp, xl_partition -t, xl_cover -e 30 -u 200, xl_coll_ck -k. These commands are recommended by the SIS package document.

Two preliminary experiments were done on the initial networks to check the effectiveness of the proposed applications of SPFDs. One was to optimize networks and the other was to count the number of connections that could be removed or replaced with other connections. In 
Table 1: Results of the optimization methods

\begin{tabular}{|r|r|r|r|r|r|r|r|r|r|r|r|}
\hline Circuits & \multicolumn{3}{|c|}{ Initial } & \multicolumn{9}{|c|}{ Area } & \multicolumn{4}{|c|}{ Level } \\
\cline { 2 - 13 } & LUT & conn & lev & LUT & conn & lev & CPU & LUT & conn & lev & CPU \\
\hline C1908 & 103 & 429 & 13 & 98 & 389 & 13 & 48.54 & 98 & 393 & 11 & 32.95 \\
\hline C432 & 66 & 275 & 17 & 63 & 257 & 16 & 12.25 & 63 & 257 & 16 & 10.03 \\
\hline alu2 & 109 & 482 & 19 & 97 & 378 & 17 & 1.75 & 97 & 378 & 17 & 1.45 \\
\hline alu4 & 208 & 862 & 24 & 192 & 723 & 26 & 21.23 & 198 & 745 & 22 & 5.5 \\
\hline apex6 & 194 & 894 & 10 & 181 & 803 & 10 & 5.23 & 181 & 803 & 10 & 4.72 \\
\hline apex7 & 73 & 292 & 6 & 67 & 247 & 11 & 1.19 & 68 & 255 & 6 & 0.78 \\
\hline cordic & 17 & 76 & 8 & 12 & 52 & 8 & 0.17 & 12 & 52 & 8 & 0.17 \\
\hline dalu & 331 & 1393 & 16 & 286 & 1115 & 10 & 15.79 & 287 & 1125 & 9 & 10.97 \\
\hline des & 1118 & 4663 & 11 & 1104 & 4407 & 22 & 3585.08 & 1111 & 4508 & 11 & 681.79 \\
\hline example2 & 105 & 451 & 5 & 100 & 396 & 8 & 4.14 & 101 & 415 & 5 & 2.04 \\
\hline frg2 & 339 & 1307 & 8 & 278 & 1019 & 9 & 22.08 & 278 & 1039 & 8 & 15.21 \\
\hline i9 & 138 & 679 & 5 & 137 & 675 & 5 & 6.88 & 137 & 675 & 5 & 4.75 \\
\hline k2 & 536 & 2325 & 9 & 528 & 2144 & 13 & 156.04 & 533 & 2267 & 9 & 19.66 \\
\hline lal & 36 & 142 & 4 & 30 & 102 & 8 & 0.46 & 31 & 121 & 3 & 0.17 \\
\hline rot & 192 & 753 & 14 & 187 & 707 & 13 & 502.3 & 187 & 707 & 13 & 409.9 \\
\hline t481 & 404 & 1738 & 21 & 379 & 1505 & 21 & 13.75 & 379 & 1505 & 21 & 11.75 \\
\hline term1 & 69 & 303 & 7 & 45 & 186 & 6 & 0.68 & 45 & 186 & 6 & 0.68 \\
\hline too_large & 188 & 882 & 12 & 179 & 805 & 12 & 36.65 & 179 & 805 & 12 & 36.65 \\
\hline tt12 & 53 & 237 & 4 & 46 & 189 & 5 & 0.28 & 47 & 196 & 4 & 0.36 \\
\hline vda & 246 & 1043 & 8 & 239 & 941 & 25 & 280.19 & 246 & 992 & 8 & 3.42 \\
\hline X1 & 111 & 455 & 6 & 96 & 383 & 7 & 2.4 & 99 & 393 & 6 & 3.19 \\
\hline x2 & 13 & 56 & 3 & 12 & 48 & 3 & 0.04 & 12 & 48 & 3 & 0.04 \\
\hline x3 & 205 & 938 & 6 & 189 & 825 & 6 & 7.47 & 189 & 830 & 5 & 3.43 \\
\hline x4 & 140 & 598 & 4 & 110 & 441 & 4 & 1.2 & 110 & 441 & 4 & 1.2 \\
\hline total & 4994 & 21273 & 240 & 4655 & 18737 & 278 & 4723.79 & 4688 & 19136 & 222 & 1260.8 \\
\hline ratio & 1.00 & 1.00 & 1.0 & 0.93 & 0.88 & 1.1 & & 0.94 & 0.90 & 0.9 & \\
\hline & & & & & & & & & &
\end{tabular}

this section, "LUT", "conn" and "lev" mean the number of LUTs, the number of connections and the number of network levels.

\subsection{Results of the Optimization Methods}

We did an experiment to check the effectiveness of the optimization method proposed in Section 4.2. Table 1 shows the results of this experiment. In Table 1, "CPU" shows the CPU run-time (sec.) on a SPARC station 20. For the procedure mentioned in Section 4.2, two kinds of heuristics were tried. In one, the output of the LUT having one fanout was selected first as $c_{i}$ in step 2; the objective was to reduce "LUT". In the other, the LUT whose number of levels was the smallest was selected as $L_{j}$ in step 4; the objective was to reduce "lev". The results are shown in the columns "Area" and "Level" in the table, respectively. The row "total" shows the total numbers of "LUT", "conn", "lev" and "CPU". The row "ratio" shows the ratios of both "Area" and "Level" to "Initial". Comparing the columns "Area" and "Level", we can observe the following. The method "Area" increases "lev" in some cases, while the method "Level" does not increase "lev". In addition, the method "Level" consumes less CPU time than the method "Area".

In the implemented method, the optimization method was applied only once and all of the SPFDs of LUTs were filtered to have one element (because of simplicity in implementing the program). Therefore, we expect that better results can be obtained if the method is applied many times and no filter is used.

\subsection{Possibility of Removing a Connection}

We did another preliminary experiment to check the effectiveness of the method proposed in Section 4.3. In the experiment, the number of connections that could be removed or replaced with other connections (called "changeable connections") was counted. The column "Num." in Table 2 shows the number of changeable connections in a network, and the column "Ratio" in Table 2 shows the ratio(\%) of changeable connections to all connections in the network. The mean value of the ratios was $73.8 \%$. The column "CPU" in Table 2 shows the CPU run-time (sec.) on a SPARC station 20 to check all connections in the network. From this experiment, we could observe that most of the connections could be removed or replaced with other connections by our method. We plan to integrate our method into routing tools and check the effectiveness of our method in the routing step.

\section{Conclusion and Future Work}

We have presented a new method to express functional permissibilities for LUT based FPGAs. The method utilizes "sets of pairs of functions" that are called SPFDs. The SPFD of an LUT (or a connection) is a set of pairs 
Table 2: The number of changeable connections

\begin{tabular}{|r|r|r|r|r|r|r|}
\hline Circuits & \multicolumn{3}{|c|}{ Initial } & Num. & Ratio & CPU \\
\cline { 2 - 4 } & LUT & conn & lev & & & \\
\hline C1908 & 103 & 429 & 13 & 357 & 83.2 & 32.7 \\
\hline C432 & 66 & 275 & 17 & 245 & 89.1 & 8.79 \\
\hline alu2 & 109 & 482 & 19 & 464 & 96.2 & 1.18 \\
\hline alu4 & 208 & 862 & 24 & 831 & 96.4 & 3.46 \\
\hline apex6 & 194 & 894 & 10 & 447 & 50.0 & 4.08 \\
\hline apex7 & 73 & 292 & 6 & 159 & 54.4 & 0.15 \\
\hline cordic & 17 & 76 & 8 & 69 & 90.7 & 0.05 \\
\hline dalu & 331 & 1393 & 16 & 1335 & 95.8 & 7.8 \\
\hline des & 1118 & 4663 & 11 & 3780 & 81.1 & 422.32 \\
\hline example2 & 105 & 451 & 5 & 171 & 37.9 & 1.31 \\
\hline frg2 & 339 & 1307 & 8 & 883 & 67.5 & 11.89 \\
\hline i9 & 138 & 679 & 5 & 364 & 53.6 & 3.43 \\
\hline k2 & 536 & 2325 & 9 & 2184 & 93.9 & 13.17 \\
\hline lal & 36 & 142 & 4 & 77 & 54.2 & 0.06 \\
\hline rot & 192 & 753 & 14 & 380 & 50.4 & 334.48 \\
\hline t481 & 404 & 1738 & 21 & 1735 & 99.8 & 10.56 \\
\hline term1 & 69 & 303 & 7 & 268 & 88.4 & 0.17 \\
\hline too_large & 188 & 882 & 12 & 868 & 98.4 & 32.01 \\
\hline ttt2 & 53 & 237 & 4 & 148 & 62.4 & 0.1 \\
\hline vda & 246 & 1043 & 8 & 917 & 87.9 & 2.35 \\
\hline x1 & 111 & 455 & 6 & 345 & 75.8 & 2.48 \\
\hline x2 & 13 & 56 & 3 & 30 & 53.5 & 0.02 \\
\hline x3 & 205 & 938 & 6 & 497 & 52.9 & 1.97 \\
\hline x4 & 140 & 598 & 4 & 342 & 57.1 & 0.4 \\
\hline & & & & & & \\
\hline
\end{tabular}

of functions that must be distinguished by the function realized at the LUT (or the connection). SPFDs make good use of properties of LUTs such that their internal logics can be changed. We have also proposed applications of SPFDs and presented preliminary experimental results to show the effectiveness of SPFDs. SPFDs used in large networks could not be calculated because of limited BDD power. Therefore, we plan to treat larger networks by methods such as network division. We also plan to integrate the method of removing connections into routing tools and check the effectiveness of SPFDs in the routing step.

\section{References}

[1] S. D. Brown, R. J. Francis, J. Rose, and Z. G. Vranesic, FIELD-PROGRAMMABLE GATE ARRAYS. Kluwer Academic Publishers, 1992.

[2] R. K. Brayton, R. Rudell, A. Sangiovanni-Vincentelli, and A. R. Wang, "MIS: A Multiple-Level Logic Optimization System," IEEE Trans. Computer-Aided Design, vol. CAD-6, pp. 1062-1081, Nov. 1987.

[3] S. Muroga, Y. Kambayashi, H. C. Lai, and J. N. Culliney, "The Transduction Method-Design of Logic Networks Based on Permissible Functions," IEEE Trans. Computers, vol. 38, pp. 1404-1424, Oct. 1989.

[4] R. Murgai, N. Shenoy, R. K. Brayton, and A. Sangiovanni-Vincentelli, "Improved Logic Synthesis Algorithms for Table Look Up Architectures," in International Conference on CAD, pp. 564-567, Nov. 1991.
[5] R. J. Francis, J. Rose, and Z. Vranesic, "Chortle-crf: Fast Technology Mapping for Lookup Table-Based FPGAs," in 28th ACM/IEEE Design Automation Conference, pp. 227-233, June 1991.

[6] K. Karplus, "Xmap: a Technology Mapper for Tablelookup Field-Programmable Gate Arrays," in 28th ACM/IEEE Design Automation Conference, pp. 240 243, June 1991.

[7] M. Tsai, T. Hwang, and Y. Lin, "Technology Mapping for Field Programmable Gate Arrays Using Binary Decision Diagram," in Proc. of the Synthesis and Simulation Meeting and International Interchange, pp. 84-92, 1992.

[8] S. Chang and M. Marek-Sadowska, "Technology Mapping via Transformations of Function Graphs," in International Conference on Computer Design, pp. 159-162, Oct. 1992.

[9] T. Sasao, "FPGA design by generalized functional decomposition," in Logic Synthesis and Optimization (T. Sasao, ed.), pp. 233-258, Kluwer Academic Publishers, 1993.

[10] J. Cong and Y. Ding, "FlowMap: An Optimal Technology Mapping Algorithm for Delay Optimization in Lookup-Table Based FPGA Design," IEEE Transactions on Computer-Aided Design of Integrated Circuits and Systems, vol. 13, pp. 1-11, Jan. 1994.

[11] H. Sawada, T. Suyama, and A. Nagoya, "Logic Synthesis for Look-up Table Based FPGAs Using Functional Decomposition and Support Minimization," in International Conference on CAD, pp. 353-358, Nov. 1995.

[12] R. E. Bryant, "Graph-based algorithm for Boolean function manipulation," IEEE Trans. Computers, vol. C-35, pp. 667-691, Aug. 1986.

[13] K. Bartlett, R. K. Brayton, G. D. Hachtel, R. M. Jacoby, and C. R. Wang, "Multi-level Logic Minimization Using Implict Don't Cares," in International Conference on CAD, pp. 723-740, June 1988.

[14] S. Yamashita, Y. Kambayashi, and S. Muroga, "Optimization Methods for Lookup-Table-Based FPGAs Using Transduction Method," in ASP-DAC '95, pp. 353-356, Aug. 1995.

[15] H. Savoj and R. K. Brayton, "The Use of Observability and External Don't Cares for Simplification of Multi-Level Networks," in 27th ACM/IEEE Design Automation Conference, pp. 297-301, June 1990.

[16] S. Yang, Logic synthesis and optimization benchmarks user guide version 3.0. MCNC, Jan. 1991. 